\title{
碘对金纳米棒的融合作用及其在四环素类抗菌素 分析测定中的应用
}

\author{
王健，吴吴，黄承志 ${ }^{*}$ \\ 发光与实时分析教育部重点实验室, 西南大学化学化工学院 药学院, 重庆 400715 \\ * 联系人, E-mail: chengzhi@swu.edu.cn
}

收稿日期：2008-05-17; 接受日期：2008-06-17

国家自然科学基金(批准号: 30570465)和国家杰出青年科学基金(批准号: 20425517)资助项目

\begin{abstract}
摘要应用等离子共振吸收光谱和扫描电子显微镜, 观察了碘和盐酸四环素反应 引起的金纳米棒形态的变化. 实验表明, 单质碘能对金纳米棒产生融合作用, 引起 金纳米棒径向比的减小和纵向吸收波长的蓝移; 但当盐酸四环素存在时, 单质碘与 盐酸四环素作用, 减低了碘的有效浓度, 减弱了碘对金纳米棒的融合作用, 使金纳 米棒的纵向吸收峰随盐酸四环素浓度的增大发生线性红移. 据此本文建立了一种测 定盐酸四环素的方法. 方法的线性范围为 $5.0 \times 10^{-5} \mathrm{~mol} / \mathrm{L} \sim 5.0 \times 10^{-4} \mathrm{~mol} / \mathrm{L}$, 检测限为 $2.4 \times 10^{-6} \mathrm{~mol} / \mathrm{L}(3 \sigma / \mathrm{k})$. 常见物质不干扰测定. 方法成功应用于合成样中四环素测定, 回收率在 $92.8 \%$ 107.2\%之间, RSD 值小于 4.3\%. 用标准加入法测定了 3 个乳制品 厂生产的牛奶中盐酸四环素, 表明牛奶中的四环素残余物浓度较低, 符合安全标准.
\end{abstract}

\section{关键词}

金纳米棒

碘

盐酸四环素

等离子共振吸收光谱
四环素类抗菌素由于能够抵抗多种病原体, 在

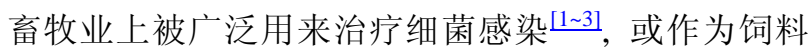
添加剂 ${ }^{[4,5]}$; 在医学上用来治疗胆囊疾病 ${ }^{[6,7]}$ 、恶性胸 膜渗透 ${ }^{[8 \sim 12]}$ 以及齿龈疾病 13$]$ 等. 但是大量使用抗生素 会引起细菌的耐药性 $[14,15]$, 以及引起某些人群的过 敏反应 ${ }^{[16]}$, 而且其降解产物亦有生物毒性 ${ }^{[17]}$. 四环 素易与 $\mathrm{Ca}^{2+}$ 结合 118$]$, 导致长期食用含有四环素类添加 剂饲料的动物骨骼中的四环素残余物含量较大 $[19]$, 鱼 ${ }^{[4,20]}$ 、肉类 ${ }^{[21 ~ 23]}$ 和蛋类 ${ }^{[24 ~ 27]}$ 以及牛奶 ${ }^{[28,29]}$ 中亦有一 定的残留. 因此四环素类的测定与人类的生活息息 相关, 具有重要意义.

四环素残余物的传统测定方法有微生物抑制实 验 ${ }^{[30]}$ 、免疫法 ${ }^{[31]}$ 、苂光法 ${ }^{[32]}$ 、毛细管电泳法 ${ }^{[20]}$ 和色 谱法 ${ }^{[33 ~ 35]}$. 这些方法提供了四环素的结构信息和药
理作用，但同时存有一定的局限性：如微生物抑制实 验周期较长, 不够灵敏; 免疫法能够提供较好的灵敏 度和选择性, 但是操作复杂, 耗时较长; 而毛细管电 泳法的重现性不好; 色谱法对四环素的特异性和灵 敏度较高, 但是需要价格昂贵的仪器设备, 样品萃取 操作繁琐, 需要专职人员操作, 限制了方法的应用. 因此需要发展一种快速、简便测定四环素的方法.

我们的前期研究表明 [36], 单质碘能够融合金纳 米棒( $\mathrm{Au}-\mathrm{NRs})$, 使金纳米棒的纵向吸收峰蓝移. 而盐 酸四环素(TC)具有酚基结构(结构式如图 1(a)), 与 $I_{2}$ 既可以氧化还原反应, 又可以发生取代反应, 从而降 低 $\mathrm{I}_{2}$ 的有效浓度, 导致金纳米棒的纵向吸收波长随着 $\mathrm{TC}$ 浓度的增大而发生线性红移. 根据金纳米棒的纵 向吸收波长与 TC浓度之间的关系, 本文建立了一种 


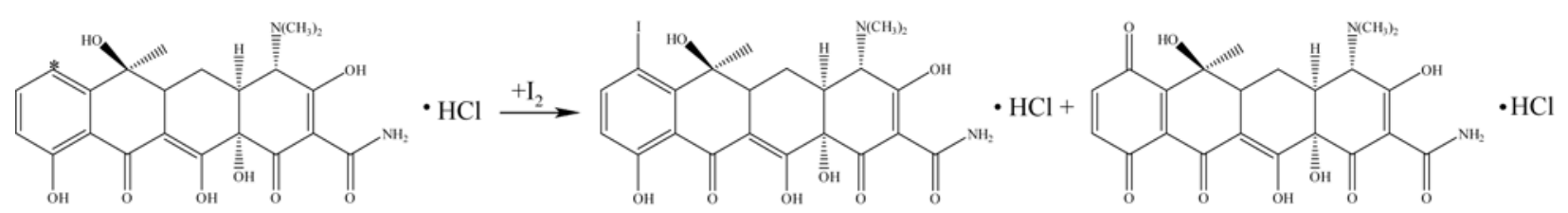

(a)

(b)

图 1 盐酸四环素与碘作用机理推测

(a) 盐酸四环素; (b) 盐酸四环素与碘反应的主要产物

测定 TC 的方法.

\section{1 实验部分}

\section{1 仪器与试剂}

F-2500 苂光分光光度计(日本日立公司), 用于测 定苂光光谱及其强度; U-3010 紫外-可见分光光度计 (日本日立公司), 用来测定等离子共振吸收光谱及强 度; S-4800 扫描电子显微镜(日本日立公司), 用来表 征金纳米粒子的大小以及形态; Spectrum GX 傅立叶 转换红外光谱仪(美国 Perkin Elmer 公司)用来测定红 外光谱; $300 \mathrm{MHz}$ 的 AV 300 核磁共振仪(瑞士 Brucker 公司)用于测定样品的核磁共振图谱; API 2000 LC/MS/MS 液质联用仪(美国 Perkin Elmer 公司)用来 测定样品的质谱; CHI 660 B 电化学工作站(上海辰华 仪器公司)用来测定溶液的循环伏安电极电位; PHS-3C 酸度计(上海大中分析仪器厂) 用来测溶液的 酸度; H1650-W 离心机(湘仪离心仪器有限公司)用于 离心以除去十六烷基三甲基溴化铵(CTAB); QL-901 旋涡混合器(海门市其林贝尔仪器制造有限公司), 用 来混合液体.

使用 $2.0 \times 10^{-3} \mathrm{~mol} / \mathrm{L}$ 氯金酸 $\left(\mathrm{HAuCl}_{4} \cdot 4 \mathrm{H}_{2} \mathrm{O}\right), 0.01$ $\mathrm{mol} / \mathrm{L}$ 嗍氢化钠, $0.10 \mathrm{~mol} / \mathrm{L}$ 抗坏血酸 $(\mathrm{L}-\mathrm{AA}), 0.20$

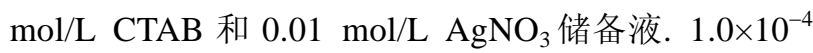
$\mathrm{mol} / \mathrm{L} \mathrm{I}$ 溶液和 $1.0 \times 10^{-3} \mathrm{~mol} / \mathrm{L} \mathrm{TC}$ 分别置于 $4^{\circ} \mathrm{C}$ 冰箱 避光保存. 使用 $\mathrm{pH} 2.15$ 盐酸-乙酸钠缓冲溶液和 1.0 $\mathrm{mol} / \mathrm{L}$ 氯化钠控制酸度和离子强度.

\section{2 样品制备}

（1）晶种生长法制备金纳米棒 [37] 使用冰冻的 $1.0 \times 10^{-3} \mathrm{~mol} / \mathrm{L} \mathrm{NaBH}_{4}$ 在 $0.087 \mathrm{~mol} / \mathrm{L} \mathrm{CTAB}$ 溶液中还 原 $2.9 \times 10^{-4} \mathrm{~mol} / \mathrm{L} \mathrm{HAuCl} \cdot 4 \mathrm{H}_{2} \mathrm{O}$ 溶液, 剧烈振动 $2 \mathrm{~min}$ 后, 溶液由橙色变为淡棕色. 该溶液于 $25^{\circ} \mathrm{C}$ 恒温水浴
锅中静置 2 24 h后形成粒径大约 $4 \mathrm{~nm}$ 的金种. 然后 在含有 $5.0 \mathrm{~mL} 2.0 \times 10^{-3} \mathrm{~mol} / \mathrm{L} \mathrm{HAuCl}_{4} \cdot 4 \mathrm{H}_{2} \mathrm{O}$ 和 7.70 $\mathrm{mL} \mathrm{H}_{2} \mathrm{O}$ 的生长液中加入 $11.88 \mathrm{~mL} 0.2 \mathrm{~mol} / \mathrm{L}$ CTAB, 溶液迅速由淡黄色变成橙色. 加入 $0.15 \mathrm{~mL} 0.01$ $\mathrm{mol} / \mathrm{L} \mathrm{AgNO}_{3}$, 摇匀, 再加入 $0.16 \mathrm{~mL} 0.1 \mathrm{~mol} / \mathrm{L} \mathrm{L}-\mathrm{AA}$, 混合 $2 \mathrm{~min}$, 溶液立即变成无色. 加入 $0.50 \mathrm{~mL}$ 上述已 静置 $2 \mathrm{~h}$ 的金种, 振荡 $20 \mathrm{~s}$ 后溶液由无色逐渐变成红 色. 将混合液放入 $25^{\circ} \mathrm{C}$ 恒温水浴锅中静置 $24 \mathrm{~h}$ 后, 形 成了径向比为 3.7 的金纳米棒.

(2) SEM 电子显微镜扫描样品的处理 为去除 CTAB 对 SEM 带来的背景干扰, 将金纳米棒溶液 1 $\mathrm{mL}$ 于 $25^{\circ} \mathrm{C}$ 以 $10000 \mathrm{rpm}$ 的转速离心 $25 \mathrm{~min}$, 弃去上 清液, 将下层残余物重新溶解于水中. 重复以上操作 后取 $5.0 \mu \mathrm{L}$ 滴于铝箔之上, 烘干. 测定电压为 $20.0 \mathrm{kV}$, 放大 10 万倍观测金纳米棒的形态变化.

(3) 实际样品的预处理 参考文献[28]将样品置于 $80^{\circ} \mathrm{C}$ 加热 $10 \mathrm{~min}$, 使蛋白变性, 离心除掉蛋白和油脂; 加热浓缩 10 倍, 用孔径为 $0.45 \mu \mathrm{m}$ 的滤膜抽滤至清.

\section{3 实验方法与步骤}

(1) 在 $1.5 \mathrm{~mL}$ 塑料管中加入 $0.10 \mathrm{~mL} \mathrm{HCl}-\mathrm{NaAc}$ 缓冲溶液 $(\mathrm{pH}$ 2.15), $0.10 \mathrm{~mL} 1.0 \mathrm{~mol} / \mathrm{L} \mathrm{NaCl}$, 摇匀; 加入一定量的 $\mathrm{I}_{2}$ 和 $\mathrm{TC}$, 摇匀, 用二次蒸馏水定容至 $0.80 \mathrm{~mL}$ ，摇匀; 在 $37^{\circ} \mathrm{C}$ 下反应 $40 \mathrm{~min}$; 然后加入 0.20 $\mathrm{mL}$ 金纳米棒溶液, 摇匀, 在 $37^{\circ} \mathrm{C}$ 下反应 $30 \mathrm{~min}$. 在 U-3010 紫外-可见分光光度计上扫描获得吸收光谱, 波长范围为 $450 \sim 850 \mathrm{~nm}$, 狭缝为 $0.5 \mathrm{~nm}$.

(2) 红外光谱的测定: 将固体样品用 $\mathrm{KBr}$ 压片, 然后测定红外光谱; 核磁共振图谱的测定, 将固体样 品溶于 $\mathrm{CD}_{3} \mathrm{COCD}_{3}$ 测定样品的 ${ }^{1} \mathrm{H} \mathrm{NMR}$; 质谱的测定： 将固体样品溶于 $50 \%(V / V)$ 丙酮-水溶液, 测定其质谱.

(3) 循环伏安(CV)电极电位的测定: 将用 $\mathrm{Al}_{2} \mathrm{O}_{3}$ 
抛光的铂碳电极插入 $\mathrm{TC}$ 和 $\mathrm{I}_{2}$ 溶液中测定其电极电位.

\section{2 结果与讨论}

\subsection{TC 与 $I_{2}$ 的作用机理探讨}

图 2 是以 $406 \mathrm{~nm}$ 激发 TC 所获得的荧光光谱, 最 大发射波长位于 $545 \mathrm{~nm}$, 在 $496.5 \mathrm{~nm}$ 处有一肩峰. 加入 $\mathrm{I}_{2}$ 后, TC 的特征苂光强度大大降低, 并且使得 $496.5 \mathrm{~nm}$ 的肩峰成为特征发射峰. 可能原因是 $\mathrm{I}_{2}$ 破坏 了 TC 的结构, 使其最大发射峰发生蓝移, 其可能的 机理如图 1 所示.

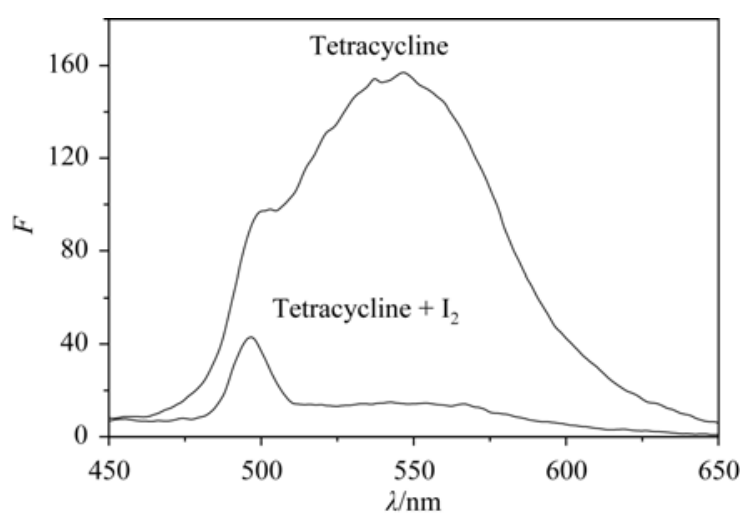

图 2 盐酸四环素与碘作用的荧光光谱

$c_{\mathrm{TC}}, 2.5 \times 10^{-4} \mathrm{~mol} / \mathrm{L} ; c_{\mathrm{I} 2}, 1.0 \times 10^{-5} \mathrm{~mol} / \mathrm{L} ; c_{\mathrm{NaCl}}, 0.10 \mathrm{~mol} / \mathrm{L} ; \mathrm{pH} 2.15$

TC 与 $\mathrm{I}_{2}$ 之间发生的反应可以通过 IR、 ${ }^{1} \mathrm{H} N M R$ 、 MS 和 $\mathrm{CV}$ 电极电位变化得到证实. 如图 3 所示,

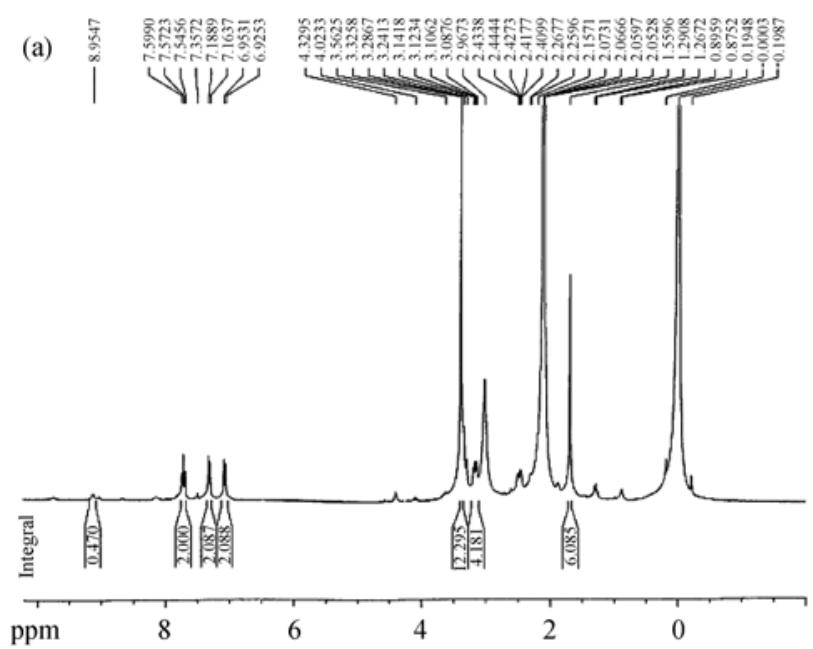

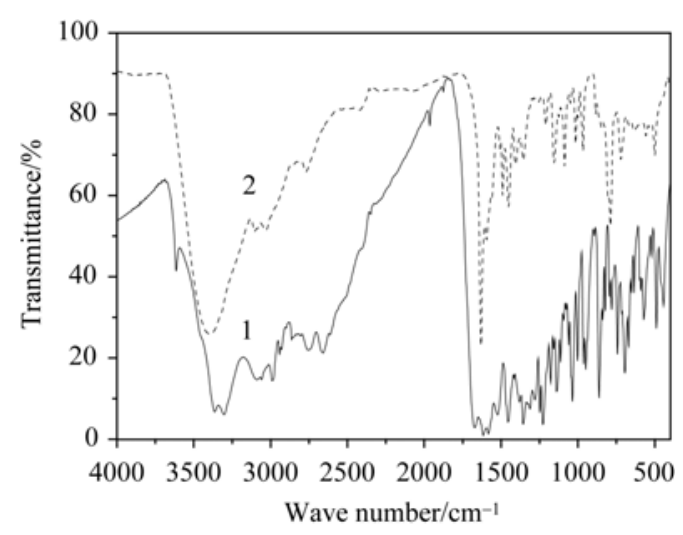

图 3 盐酸四环素(1)和碘作用后(2)的红外吸收光谱

$\mathrm{TC}$ (曲线 1)3650 $\mathrm{cm}^{-1}$ 处的酚羟基伸缩振动峰在与 $\mathrm{I}_{2}$ 反应后消失(曲线 2), 而 $1690 \mathrm{~cm}^{-1}$ 的红外强度明显增 强, 说明有新的 $\mathrm{C}=\mathrm{O}$ 生成. 此外, $500 \mathrm{~cm}^{-1}$ 处有一 $\mathrm{C}$ - $\mathrm{I}$ 伸缩振动. 因此推测, $\mathrm{TC}$ 与 $\mathrm{I}_{2}$ 之间既可以发生氧 化还原反应, 又可以发生取代反应.

核磁共振图谱(图 4)显示, $6.95 \mathrm{ppm}$ 处的化学位 移消失, 说明 $\mathrm{C}_{7}$ 上的 $\mathrm{H}($ *标示处)消失, 推测可能发 生了取代反应或者氧化还原反应. 同时，由于-I 的诱 导效应，使邻位 $\mathrm{H}$ 的化学位移移向低场，因此说明 $\mathrm{TC}$ 与 $\mathrm{I}_{2}$ 发生了取代反应; 此外, 在 $6.80 \mathrm{ppm}$ 附近出 现了较弱的 ${ }^{1} \mathrm{H}$ NMR 信号, 符合醌式结构的 ${ }^{1} \mathrm{H}$ NMR, 说明 $\mathrm{TC}$ 与 $\mathrm{I}_{2}$ 反应有醌式结构生成. 由此可以看出, $\mathrm{TC}$ 与 $\mathrm{I}_{2}$ 的反应产物是取代产物与氧化产物的混合物.

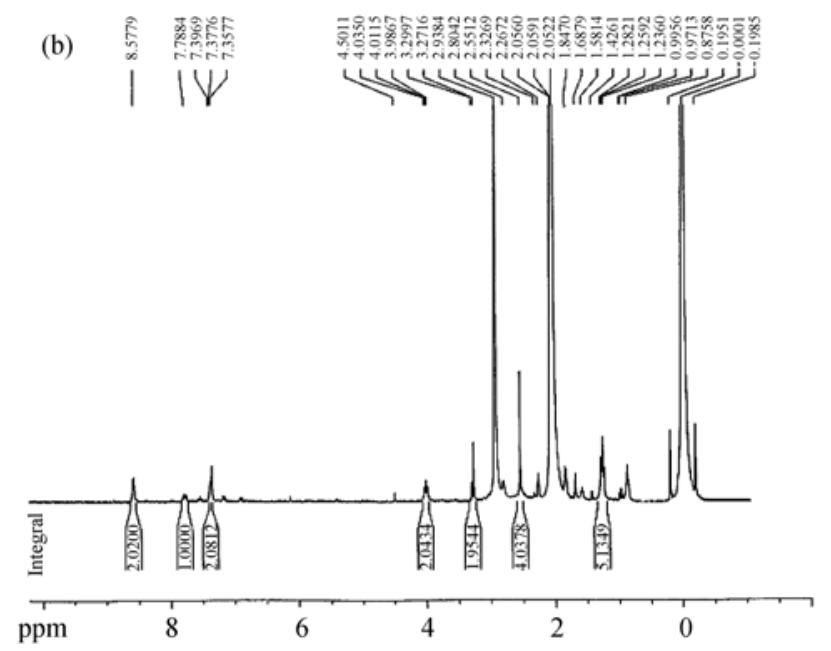

图 4 盐酸四环素(a)与碘作用 $(b)$ 的核磁共振图谱

$c_{\mathrm{TC}}, 2.5 \times 10^{-4} \mathrm{~mol} / \mathrm{L} ; c_{12}, 1.0 \times 10^{-5} \mathrm{~mol} / \mathrm{L}$; 溶剂: $\mathrm{CD}_{3} \mathrm{COCD}_{3}$ 

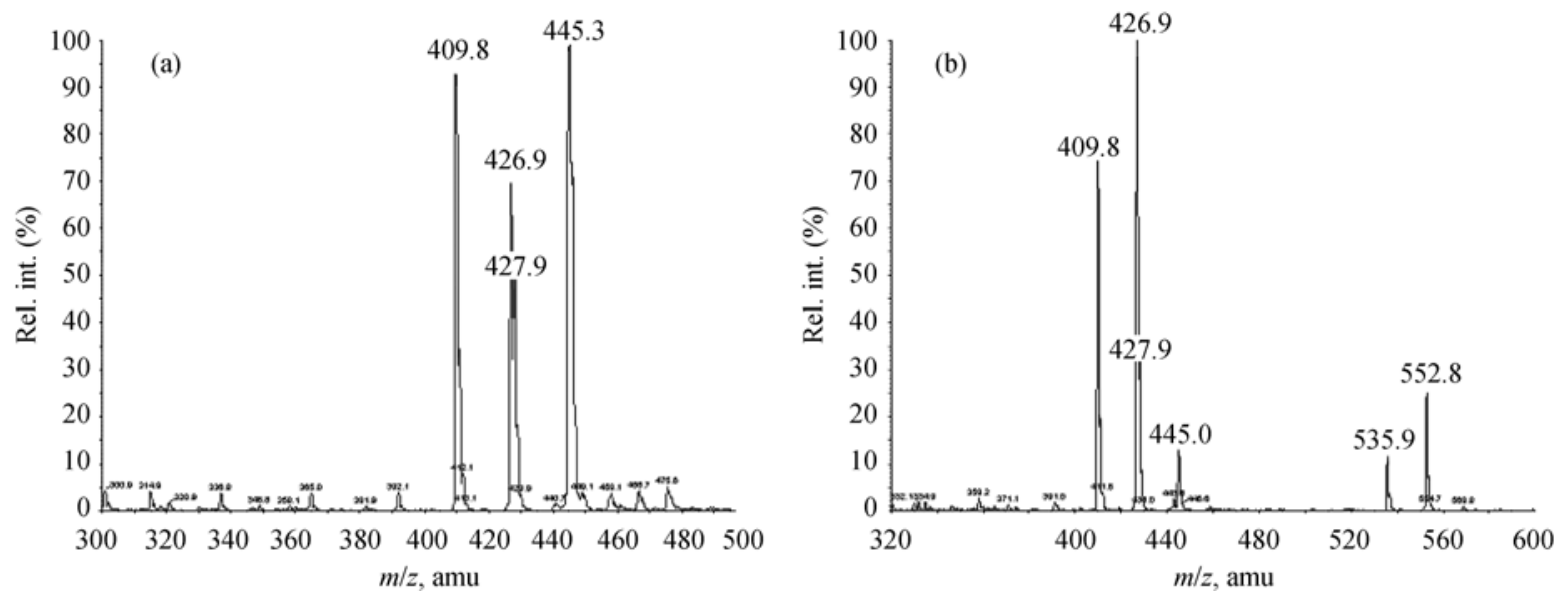

\section{图 5 盐酸四环素 $(a)$ 与碘作用(b)的质谱}

$c_{\mathrm{TC}}, 2.5 \times 10^{-4} \mathrm{~mol} / \mathrm{L} ; c_{\mathrm{I} 2}, 1.0 \times 10^{-5} \mathrm{~mol} / \mathrm{L}$; 溶剂: $50 \%(V / V)$ 丙酮-水溶液

$\mathrm{TC}$ 的分子量为 480.9 , 反应前的主要丰度表现为 核质比为 445.3 的碎片峰, 即 TC 去掉 $\mathrm{Cl}^{-}$, 然后分别 裂解掉 $\mathrm{OH} 、 \mathrm{H} 、 \mathrm{OH}$ 得到了核质比为 427.9, 426.9, 409.8 的碎片峰; 与 $\mathrm{I}_{2}$ 反应后, 由于 $\mathrm{TC}$ 的氧化产物的 生成使 426.9 处的碎片峰的丰度增大，同时可以看出， 在 552.8 和 535.9 处出现新的碎片峰, 分别由 (426.9+126.9-1)和(409.8+127.1-1)得到, 即可以证明 $\mathrm{I}_{2}$ 取代了 $\mathrm{TC}$ 上的 $\mathrm{H}$ (图 5).

此外, TC与 $\mathrm{I}_{2}$ 的氧化还原反应还可以通过循环伏 安电化学方法得到证实. 从图 6 可以看出, $\mathrm{TC}$ 和 $\mathrm{I}_{2}$ 都 没有明显的氧化还原电位, 但是两者反应后出现了 明显的不对称电位，说明该反应是不可逆反应，可自 发进行. 从而可以说明 TC可以作为还原剂被氧化剂 氧化 ${ }^{[38]}$.

\section{2 盐酸四环素的测定}

\subsection{1 实验原理}

因为 $\mathrm{I}_{2}$ 能够融合金纳米棒, 缩短金纳米棒的径向

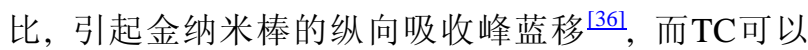
与 $\mathrm{I}_{2}$ 作用, 使 $\mathrm{I}_{2}$ 的有效浓度降低, 进而减弱了 $\mathrm{I}_{2}$ 对金 纳米棒的融合作用. 所以如图 7 所示, 当体系中存在 金纳米棒和 $\mathrm{I}_{2}$ 时, 随着TC浓度的增大, 金纳米棒的纵 向吸收波长逐渐红移, 并且吸光度逐渐增强.

通过 SEM 可以看出，单独的金纳米棒是分散的 (图 8(a)), 并且具有较好的棒状结构, 但当加入 $I_{2}$ 后, 金纳米棒逐渐融合变为球形, 粒径变大; 径向比减小

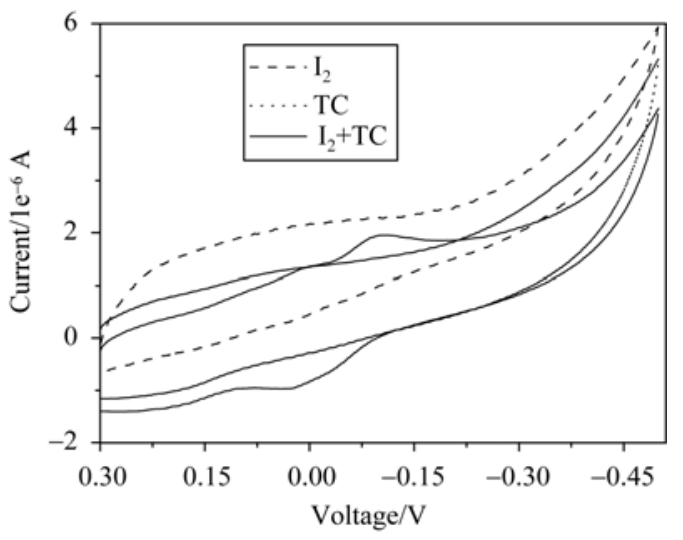

图 6 盐酸四环素与碘作用的循环伏安图

$c_{\mathrm{TC}}, 2.5 \times 10^{-4} \mathrm{~mol} / \mathrm{L} ; c_{\mathrm{I} 2}, 1.0 \times 10^{-5} \mathrm{~mol} / \mathrm{L} ; c_{\mathrm{NaCl}}, 0.10 \mathrm{~mol} / \mathrm{L} ; \mathrm{pH} 2.15$

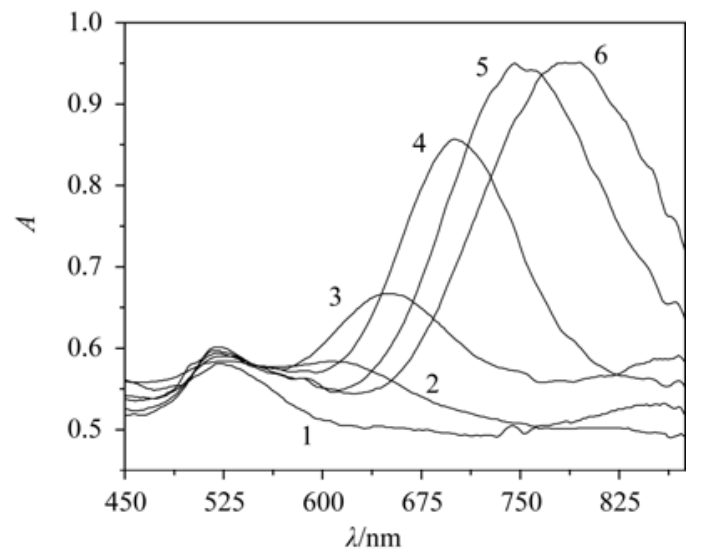

图 7 金纳米棒与 $\mathbf{I}_{2}$ 和盐酸四环素作用后的吸收光谱

$c_{\mathrm{Au} \mathrm{NRs}}, 8.0 \times 10^{-5} \mathrm{~mol} / \mathrm{L} ; c_{\mathrm{I} 2}, 1.0 \times 10^{-5} \mathrm{~mol} / \mathrm{L} ; c_{\mathrm{NaCl}}, 0.10 \mathrm{~mol} / \mathrm{L} ; \mathrm{pH}$ 2.15. $c_{\mathrm{TC}}\left(\times 10^{-4} \mathrm{~mol} / \mathrm{L}\right), 1,0 ; 2,1.0 ; 3,2.0 ; 4,3.0 ; 5,4.0 ; 6,5.0$ 

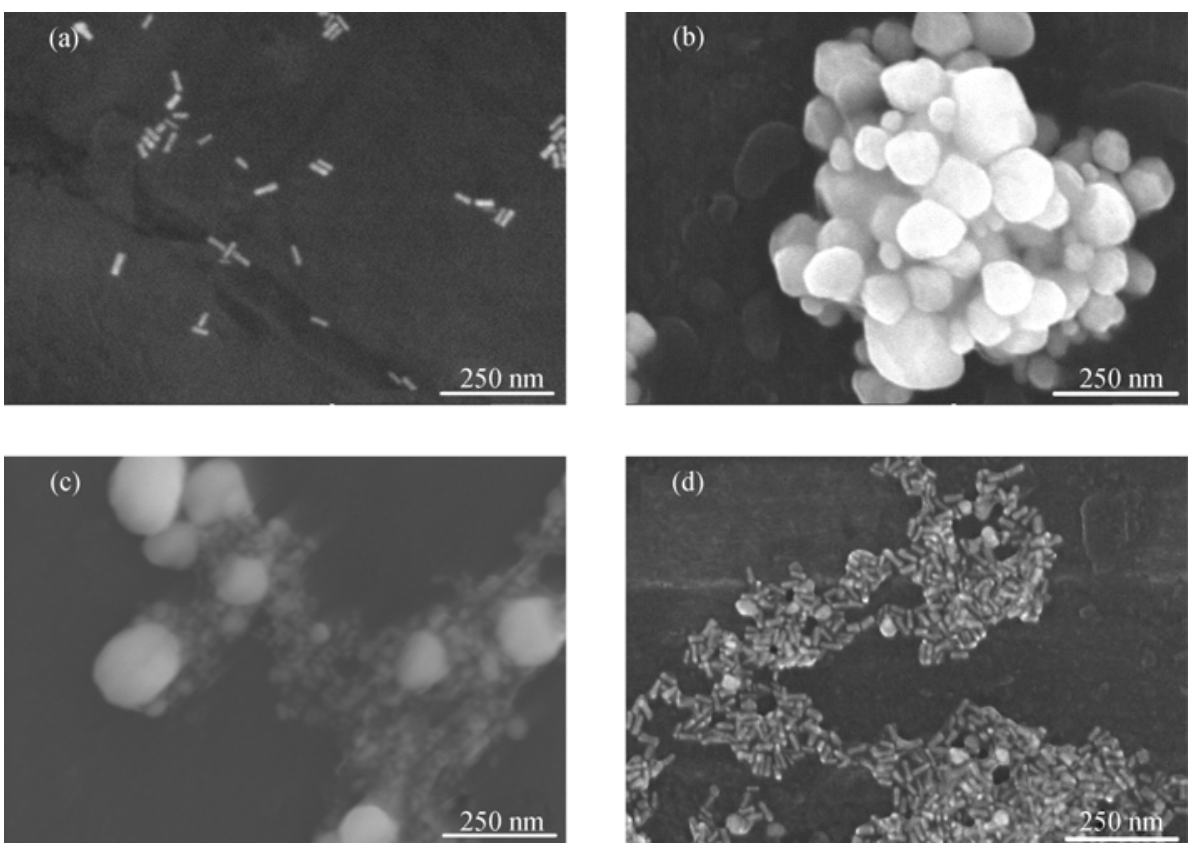

图 8 金纳米棒加入碘和盐酸四环素的 SEM 图

(a) Au NRs; (b) Au NRs $+\mathrm{I}_{2}$; (c)和(d) Au NRs $+\mathrm{I}_{2}+\mathrm{TC}$. $c_{\mathrm{Au} \mathrm{NRs}}, 8.0 \times 10^{-5} \mathrm{~mol} / \mathrm{L} ; c_{\mathrm{TC}}\left(\times 10^{-4} \mathrm{~mol} / \mathrm{L}\right), 2.5 \mathrm{in}(\mathrm{c}), 5.0 \mathrm{in}(\mathrm{d}) ; c_{\mathrm{I} 2}, 1.0 \times 10^{-5} \mathrm{~mol} / \mathrm{L} ; c_{\mathrm{NaCl}}$, $0.10 \mathrm{~mol} / \mathrm{L} ; \mathrm{pH} 2.15$

(图 8(b)); 在 $\mathrm{I}_{2}$ 溶液中加入 $\mathrm{TC}$, 然后再加入金纳米棒, 则球形纳米粒子的粒径越来越小, 并逐渐出现棒状 结构, 但是仍有一定的球状物(图 8(c), (d)). 这与吸收 图谱的变化趋势相吻合.

\subsection{2 实验条件的优化}

实验结果表明, 由于溶液中大量 CTAB的存在, $\mathrm{NaCl}$ 的浓度对体系的影响较小. 为了提高体系的抗 干扰能力, 所以在反应体系中加入 $0.1 \mathrm{~mol} / \mathrm{L} \mathrm{NaCl}$ 来 控制体系的离子强度. 由于在强酸性溶液中, 四环素 不稳定 ${ }^{[39,40]}$, 且 $\mathrm{I}_{2}$ 在碱性条件下发生歧化反应, 所以 选择酸性缓冲溶液( $\mathrm{pH}$ 0.65 5.20). 不加入 TC 时, 金 纳米棒的纵向吸收波长随酸度的降低逐渐红移, 可 能原因是降低酸度不利于 $I_{2}$ 对金纳米棒发生作用，而 加入 $\mathrm{TC}$ 后, 金纳米棒的纵向吸收波长先蓝移后红移, 说明酸度的降低不利于 $\mathrm{TC}$ 与 $\mathrm{I}_{2}$ 之间反应的进行. 而 后来的红移则归因于在弱酸条件下不利于 $I_{2}$ 对金纳 米棒发生融合作用.

由图 9 可以看出, 随 $\mathrm{I}_{2}$ 与 $\mathrm{TC}$ 反应时间的延长, 金 纳米棒的纵向吸收峰逐渐红移, 并在 $30 \mathrm{~min}$ 内达到 平衡, 说明 $\mathrm{I}_{2}$ 与 $\mathrm{TC}$ 的反应为一动态变化过程. 所以,
为保证反应完全, 我们先将 $\mathrm{I}_{2}$ 与 $\mathrm{TC}$ 反应 $40 \mathrm{~min}$, 然后 加入金纳米棒反应 $30 \mathrm{~min}$ 后测定 ${ }^{[36]}$.

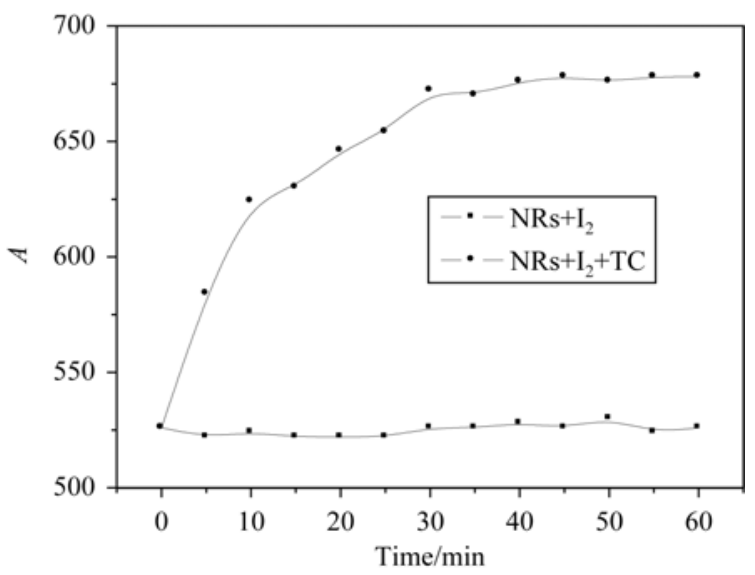

图 9 时间对金纳米棒纵向吸收峰波长的影响

$c_{\mathrm{Au} \mathrm{NRs}}, 8.0 \times 10^{-5} \mathrm{~mol} / \mathrm{L} ; c_{\mathrm{TC}}, 2.5 \times 10^{-4} \mathrm{~mol} / \mathrm{L} ; c_{12}, 1.0 \times 10^{-5} \mathrm{~mol} / \mathrm{L}, c_{\mathrm{NaCl}}$, $0.10 \mathrm{~mol} / \mathrm{L} ; \mathrm{pH} 2.15$

\subsection{3 共存物质的影响}

实验考察了金属离子、药物、氨基酸和蛋白质等 各种共存物质的影响, 结果列于表 1 中. 多数物质能 
表 1 共存物质的影响 ${ }^{\mathrm{a})}$

\begin{tabular}{|c|c|c|c|c|c|}
\hline 干扰物质 & 容许量/mg·mL ${ }^{-1}$ & 相对误差/\% & 干扰物质 & 容许量 $/ 10^{-4} \mathrm{~mol} \cdot \mathrm{L}^{-1}$ & 相对误差/\% \\
\hline 谷氨酸 & 0.25 & +2.9 & 赖氨酸 & 25 & +4.0 \\
\hline 半胱氨酸 & 0.25 & +5.6 & 丙氨酸 & 25 & -9.2 \\
\hline HSA & 0.25 & +1.5 & 盐酸土霉素 & 2.5 & +1.5 \\
\hline BSA & 0.25 & +1.8 & 安乃近 & 2.5 & -0.5 \\
\hline 刀豆蛋白 & 0.25 & -0.3 & 头孢噻肜 & 2.5 & +0.8 \\
\hline 胃蛋白酶 & 0.25 & +1.8 & 头孢唑啉 & 2.5 & +1.0 \\
\hline 蜗牛酶 & 0.25 & +0.6 & 阿魏酸 & 2.5 & +0.5 \\
\hline$\alpha$-糜蛋白酶 & 0.25 & +5.4 & 链霉素 & 2.5 & +0.2 \\
\hline 凝血酶 & 0.25 & +5.4 & 卡那霉素 & 2.5 & -7.5 \\
\hline fsDNA & 1.2 & +0.2 & 环丙沙星 & 1.0 & -6.1 \\
\hline ctDNA & 1.9 & +6.1 & $\mathrm{Zn}^{2+}, \mathrm{SO}_{4}^{2-}$ & 250 & +2.3 \\
\hline 葡萄糖 & 49 & +7.3 & $\mathrm{Mg}^{2+}, \mathrm{Cl}^{-}$ & 250 & +5.1 \\
\hline 蔗糖 & 85 & +7.8 & $\mathrm{Cu}^{2+}, \mathrm{Cl}^{-}$ & 250 & +4.8 \\
\hline 乳糖 & 90 & +7.3 & $\mathrm{Ba}^{2+}, \mathrm{Cl}^{-}$ & 250 & +6.7 \\
\hline 麦芽糖 & 90 & +7.3 & $\mathrm{~K}^{+}, \mathrm{Cl}^{-}$ & 250 & +7.5 \\
\hline
\end{tabular}

a) $c_{\mathrm{Au} \mathrm{NRs}}, 8.0 \times 10^{-5} \mathrm{~mol} / \mathrm{L} ; c_{\mathrm{TC}}, 2.5 \times 10^{-4} \mathrm{~mol} / \mathrm{L} ; c_{\mathrm{I} 2}, 1.0 \times 10^{-5} \mathrm{~mol} / \mathrm{L} ; c_{\mathrm{NaCl}}, 0.10 \mathrm{~mol} / \mathrm{L} ; \mathrm{pH} 2.15$

够在较高的浓度下对 TC 的测定不产生干扰，但是环 丙沙星的容许浓度较低, 这是因为环丙沙星具有氧 化性，氧化了一部分 TC，降低了 TC 的有效浓度，所 以可以直接检测简单的样品中的 TC, 而对于复杂样 品, 可加入重水消除环丙沙星的影响.

\subsection{4 分析参数}

实验发现, 金纳米棒的纵向吸收峰的波长与 $\mathrm{TC}$ 的浓度在 $5.0 \times 10^{-5} \mathrm{~mol} / \mathrm{L} \sim 5.0 \times 10^{-4} \mathrm{~mol} / \mathrm{L}$ 范围内成
线性关系, 线性方程为: $\lambda=539.3+52 c\left(10^{-4} \mathrm{~mol} / \mathrm{L}, r\right.$ $=0.9978)$, 检测限为 $2.4 \times 10^{-6} \mathrm{~mol} / \mathrm{L}(3 \sigma / \mathrm{k})$. 这是对 TC 进行定量测定的依据.

该方法可以应用于合成样中 TC 的测定. 主要考 察了各种氨基酸、蛋白质和 DNA 以及金属离子对 TC 测定的影响. 结果列于表 2 中. 实验结果表明, TC 的 回收率在 92.8\% 107.2\%之间, RSD 值小于 4.3\%. 表 明该条件下, 共存离子对体系干扰较小, 该方法可用 于实际样品的测定.

表 2 合成样的测定 ${ }^{a)}$

\begin{tabular}{cccccc}
\hline 样品 & 加入浓度 $/ 10^{-5} \mathrm{~mol} \cdot \mathrm{L}^{-1}$ & 各合成样组分 & 回收浓度 $/ 10^{-5} \mathrm{~mol} \cdot \mathrm{L}^{-1}$ & 回收率 $/ \%$ & 相对偏差 $/ \%(n=5)$ \\
\hline 1 & 5.00 & 葡萄糖, 麦芽糖, $\mathrm{KCl}$, 半胱氨酸 & $4.92 ~ 5.36$ & $92.8 \sim 107.2$ & 4.3 \\
2 & 7.50 & 溶菌酶, 链霸素, fsDNA, $\mathrm{MgCl}_{2}$. & $7.07 \sim 7.63$ & $94.3 \sim 101.7$ & 3.7 \\
3 & 10.0 & $\mathrm{ZnSO}_{4}$, 刀豆蛋白,乳糖, 胃蛋白酶. & $9.29 \sim 10.04$ & $92.9 \sim 100.4$ & 3.1 \\
\hline
\end{tabular}

a) $c_{\mathrm{NaCl}}, 0.10 \mathrm{~mol} / \mathrm{L} ; c_{\mathrm{Au} \mathrm{NRs}}, 8.0 \times 10^{-5} \mathrm{~mol} / \mathrm{L} ; c_{\mathrm{I} 2}, 1.0 \times 10^{-5} \mathrm{~mol} / \mathrm{L} ; \mathrm{pH} 2.15$.

浓度: $\left(10^{-3} \mathrm{~mol} / \mathrm{L}\right)$ 葡萄糖, 1.0 ; 麦芽糖, 1.0 ; 乳糖, 1.0 ; 链霉素, $0.01 ; \mathrm{KCl}, 1.0 ; \mathrm{MgCl}_{2}, 1.0 ; \mathrm{ZnSO}_{4}, 1.0 ;(\mu \mathrm{g} / \mathrm{mL})$ : 半胱氨酸, $1.0 ;$ 溶菌酶, 10 ; fsDNA, 50; 刀豆蛋白, 10; 胃蛋白酶, 10 
表 3 牛奶中四环素的测定 ${ }^{\text {a) }}$

\begin{tabular}{ccccc}
\hline 样品 & 加入浓度 $/ 10^{-4} \mathrm{~mol} \cdot \mathrm{L}^{-1}$ & 测得浓度 $/ 10^{-4} \mathrm{~mol} \cdot \mathrm{L}^{-1}$ & 回收率/\%, $n=5$ & $\mathrm{RSD} / \%, n=5$ \\
\hline 伊利 & 0.50 & $0.48 \sim 0.52$ & $96.0 \sim 104.0$ & 3.2 \\
蒙牛 & 1.00 & $0.97 \sim 1.05$ & $97.0 \sim 105.0$ & 3.7 \\
天友 & 1.50 & $1.46 \sim 1.57$ & $97.3 \sim 104.7$ & 3.5 \\
\hline
\end{tabular}

a) $c_{\mathrm{NaCl}}, 0.10 \mathrm{~mol} / \mathrm{L} ; c_{\mathrm{Au} \mathrm{NRs}}, 8.0 \times 10^{-5} \mathrm{~mol} / \mathrm{L} ; c_{\mathrm{TC}}, 2.5 \times 10^{-4} \mathrm{~mol} / \mathrm{L} ; c_{\mathrm{I} 2}, 1.0 \times 10^{-5} \mathrm{~mol} / \mathrm{L} ; \mathrm{pH} 2.15$

\subsection{5 样品分析}

欧洲联盟规定，为了人类安全，牛奶中四环素的 最大残余量为 $100 \mathrm{ng} / \mathrm{mL}^{[28]}$. 取三个不同厂家(内蒙 古伊利实业集团股份有限公司、内蒙古蒙牛乳业(集 团)股份有限公司、重庆市天友乳品二厂有限公司)生 产的纯牛奶, 按 1.3 的实验方法对其中的 TC的含量进 行测定，每个样品平行测定 5 次，同时进行标准加入 回收率实验, 实验结果见表 3. 实验表明：加标回
收率为 $96.0 \%$ 105.0\%, 说明牛奶中四环素残留物的 浓度很低, 可以安全饮用.

\section{3 结语}

基于 $\mathrm{I}_{2}$ 对金纳米棒的融合作用引起的金纳米棒 纵向吸收波长蓝移，而盐酸四环素降低了 $\mathrm{I}_{2}$ 浓度导致 金纳米棒纵向吸收波长的红移，建立了一种分光光 度法测定盐酸四环素的方法. 该方法简便快速.

\section{参考文献}

1 Chopra I, Roberts M. Tetracycline antibiotics: mode of action, applications, molecular biology, and epidemiology of bacterial resistance. Microbiol Mol Biol Rev, 2001, 65(2): 232-260[DOI]

2 Polacek N, Mankin A S. The ribosomal peptidyl transferase center: structure, function, evolution, inhibition. Crit Rev Biochem Mol Biol, 2005, 40(5): 285-311 [DOI]

3 Malfertheiner P, Mégraud F, O'Morain C, Hungin A P, Jones R, Axon A, Graham D Y, Tytgat G. European Helicobacter Pylori Study Group (EHPSG). Current concepts in the management of helicobacter pylori infection- the Maastricht 2-2000 Consensus Report. Aliment Pharmacol Ther, 2002, 16(2): 167-180[DOI]

4 Pellinen T, Bylund G, Virta M, Niemi A, Karp M. Detection of traces of tetracyclines from fish with a bioluminescent sensor strain incorporating bacterial luciferase reporter genes. J Agric Food Chem, 2002, 50(17): 4812 - 4815[DOI]

5 Roberts M C. Tetracycline resistance determinants: mechanisms of action, regulation of expression, genetic mobility, and distribution. FEMS Microbiol Rev, 1996, 19(1): 1-24[DOI]

6 El-Mufti M. Sclerotherapy of the human gallbladder using ethanol and tetracycline hydrochloride. Br J Surg, 1993, 80(7): 916-916[DOI]

7 Shaw D W W, Bertino R E, Mulholland M W. Use of tetracycline for sclerosis of a biliary-cutaneous fistula. AJR Am J Roentgenol, 1989, 153(1): 65-66

8 Hartman D L, Gaither J M, Kesler K A, Mylet D M, Brown J W, Mathur P N. Comparison of insufflated talc under thoracoscopic guidance with standard tetracycline and bleomycin pleurodesis for control of malignant pleural effusions. Thorac Cardiovasc Surg, 1993, 105(4): 743-747

9 Zaloznik A J, Oswald S G, Langlin M. Intrapleural tetracycline in malignant pleural effusions: a randomized study. Cancer 1983, 51(4): $752-755[\mathrm{DOI}]$

10 Bayly T C, Kisner D L, Sybert A, Macdonald J S, Tsou E, Schein P S. Tetracycline and quinacrine in the control of malignant pleural effusions: a randomized trial. Cancer, 1978, 41(3): 1188-1192[DOI]

11 Fentiman I S, Rubens R D, Haward J L. A Comparison of intracavitary talc and tetracycline for the control of pleural effusions secondary to breast cancer. Eur J Cancer Clin Oncol, 1986, 22(9): 1079-1081[DOI] 
12 Ruckdeschel J C, Moores D, Lee J Y, Einhorn L H, Mandelbaum I, Koeller J, Weiss G R, Losada M, Keller J H. Intrapleural therapy for malignant pleural effusions: a randomized comparison of bleomycin and tetracycline. Chest, 1991, 100(6): 1528 - 1535[DOI]

13 Listgarten M A, Lindhe J, Heilden L. Effect of tetracycline and/or scaling on human periodontal disease: clinical microbiological and histological observation. J Clin Periodontol, 1978, 5(4): 246-271 [DOI]

14 McManus M C. Mechanisms of bacterial resistance to antimicrobial agents. Am J Health Syst Pharm, 1997, 54(12): 1420-1433

15 Chanda P K, Ganguly T, Das M, Lee C Y, Luong T T, Sau S. Detection of antistaphylococcal and toxic compounds by biological assay systems developed with a reporter staphylococcus aureus strain harboring a heat inducible promoter - lacZ transcriptional fusion. J Biochem Mol Biol, 2007, 40(6): 936-943

16 Heeschen W H. Residues of antibiotics and sulfonamides in milk: significance and toxicological evaluation, legal situation within the European Community (EC), and method related activities of the International Dairy Federation (IDF). Bull Int Dairy Fed, 1993, 283: $3-12$

17 Halling-Sørensen B, Sengeløv G, Tjørnelund J. Toxicity of tetracyclines and tetracycline degradation products to environmentally relevant bacteria, including selected tetracycline-resistant bacteria. Arch Environ Contam Toxicol, 2002, 42(3): 263-271 [DOI]

18 Wessels J M, Ford W E, Szymczak W, Schneider S. The complexation of tetracycline and anhydrotetracycline with $\mathrm{Mg}^{2+}$ and $\mathrm{Ca}^{2+}$ : a spectroscopic study. J Phys Chem B, 1998, 102(46): 9323-9331 [DOI]

19 Kühne M, Wegmann S, Kobe A, Fries R. Tetracycline residues in bones of slaughtered animals. Food Control, 2000, 11(3): $175-180[\mathrm{DOI}]$

20 Kowalski P. Capillary electrophoretic method for the simultaneous determination of tetracycline residues in fish samples. J Pharmaceut Biomed Anal, 2008, 47(3): 487-493[DOI]

21 Honikel K O, Schmidt U, Woltersdorf W, Leistner L. Effect of storage and processing on tetracycline residues in meat and bones. J Assoc Off Anal Chem, 1978, 61(5): 1222-1227

22 Kürner U, Kühne M, Wenzel S. Tetracycline residues in meat and bone meals. Part I : methodology and examination of field samples. Food Addit Contam, 2001, 18(4): 293-302[DOI]

23 Kühne M, Körner U, Wenzel S. Tetracycline residues in meat and bone meals. Part II: the effect of heat treatments on bound tetracycline residues. Food Addit Contam, 2001, 18(7): 593-600

24 Zurhelle G, Petz M, Mueller-Seitz E, Siewert E. Metabolites of oxytetracycline, tetracycline, and chlortetracycline and their distribution in egg white, egg yolk, and hen plasma. J Agric Food Chem, 2000, 48(12): 6392-6396[DOI]

25 Roudaut B, Moretain J P, Boisseau J. Excretion of tetracycline and chlortetracycline in eggs after oral medication of laying hens. Food Addit Contam, 1989, 6(1): 71-78

26 Zurhelle G, Müller-Seitz E, Petz M. Automated residue analysis of tetracyclines and their metabolites in whole egg, egg white, egg yolk and hen's plasma utilizing a modified ASTED-system. J Chromatogr B, 2000, 739(1): 191—203[DOI]

27 Zurhelle G, Petz M, Müeller-Seitz E, Siewert E. Tetracyclines and their metabolites in egg white and egg yolk. Z Lebensm Unters Forsch A, 1999, 208(4): 299-300[DOI]

28 Nouws J M, Loeffen G, Schouten J, van Egmond H, Keukens H, Stegeman H. Testing of raw milk for tetracycline residues. J Dairy Sci, 1998, 81(9): 2341-2345

29 Aureli P, Ferrini A M, Mannoni V. Effect of some proteolytic enzymes on microbial detection levels for tetracyclines and sulphonamides in milk serum. Arch Lebensmittelhyg, 1999, 50(4-5): 115-118

30 Cheoymang A, Hudchinton D, Kioy D, Na-Bangchang K. Bioassay for determination of fosmidomycin in plasma and urine: application for pharmacokinetic dose optimization. J Microbiol Meth, 2007, 69 (1): 65-69[DOD]

31 Konda L N, Füleky G, Morovján G. Subcritical water extraction to evaluate desorption behavior of organic pesticides in soil. J Agric Food Chem, 2002, 50(8): 2338-2343[DOI]

32 Ni Y, Lin D, Kokot S. Synchronous fluorescence and UV-vis spectroscopic studies of interactions between the tetracycline antibiotic, aluminium ions and DNA with the aid of the methylene blue dye probe. Anal Chim Acta, 2008, 606(1): 19-25[DOI]

33 Hamscher G, Sczesny S, Höper H, Nau H. Determination of persistent tetracycline residues in soil fertilized with liquid manure by 
high-performance liquid chromatography with electrospray ionization tandem mass spectrometry.Anal Chem, 2002, 74(7): $1509-1518[\mathrm{DOI}]$

34 Oka H, Ito Y, Matsumoto H. Chromatographic analysis of tetracycline antibiotics in foods. J Chromatogr A, 2000, 882(1-2): $109-133[\mathrm{DOI}]$

35 Wang L, Yang H, Zhang C, Mo Y, Lu X. Determination of oxytetracycline, tetracycline and chloramphenicol antibiotics in animal feeds using subcritical water extraction and high performance liquid chromatography. Anal Chim Acta, 2008, 619(1): 54-58[DOD]

36 Wang J, Li Y F, Huang C Z. Identification of iodine-induced morphological transformation of gold nanorods. J Phys Chem C, 2008, 112(31): 11691-11695[DOI]

37 Jiang X C, Brioude A, Pileni M P. Gold nanorods: limitations on their synthesis and optical properties. Colloid Surface A, 2006, 277(1-3): 201-206[DOI]

38 胡泊, 黄承志, 张立. 基于多壁碳纳米管的盐酸四环素催化等离子体共振光散射分析. 中国科学 B 辑: 化学, 2008, 38(7): $578-583$

39 Sassman S A, Lee L. Sorption of three tetracyclines by several soils: assessing the role of $\mathrm{pH}$ and cation exchange. Environ Sci Technol, 2005, 39(19): 7452-7459[DOI]

40 Wu Y, Fassihi R. Stability of metronidazole, tetracycline $\mathrm{HCl}$ and famotidine alone and in combination. Int J Pharm, 2005, 290(1-2): $1-13 \underline{[\mathrm{DOI}]}$ 